\title{
Thermal alterations in patients with inflammatory diseases: a comparison between psoriatic and rheumatoid arthritis
}

\author{
A. Capo', J. Di Paolo', E. Celletti ${ }^{2}$, E. Ismail ${ }^{3}$, A. Merla ${ }^{3}$, P. Amerio ${ }^{1}$ \\ ${ }^{1}$ Department of Medicine and Aging Science, Dermatologic Clinic, University G. d'Annunzio, Chieti; \\ ${ }^{2}$ Department of Medicine and Aging Science, Rheumatology, G. d'Annunzio University, Chieti; \\ 3ITAB-Institute of Advanced Biomedical Technologies and Department of Neuroscience \\ and Imaging, University G. d'Annunzio, Chieti
}

\begin{abstract}
SUMMARY
Functional infrared imaging (fIRI) is used to provide information on circulation, thermal properties and thermoregulatory function of the cutaneous tissue in several clinical settings.

This study aims to evaluate the application of fIRI in rheumatoid arthritis (RA) assessment, evaluating the thermoregulatory alterations due to joint inflammation in RA patients both in basal conditions and after a mild functional (isometric) exercise, using the same protocol we projected in our recent work on psoriatic arthritis (PsA); fIRI outcomes were compared with those provided by power-Doppler ultrasonography.

Ten patients with RA and 11 healthy controls were enrolled in the study. The cutaneous temperature dynamics of 20 regions of interest located on the dominant hand were recorded by means of high-resolution thermal imaging at baseline and after a functional exercise.

RA patients showed lower thermal parameters compared to healthy controls, suggesting that the RA-related inflammatory state alters the normal thermal properties of the skin overlying inflamed joints. These results are different from PsA data observed in the previous study.

fIRI applied to the study of the response to a functional stimulus may represent an innovative, non-invasive, and operator-independent method for the assessment of early RA.
\end{abstract}

Key words: Psoriatic arthritis; Rheumatoid arthritis; Infrared imaging; Inflammatory diseases.

\section{INTRODUCTION}

Fan unctional Infrared imaging (fIRI) is a non-invasive diagnostic technique used for diagnosis and follow-up in several clinical settings (Raynaud's phenomenon, systemic sclerosis, melanoma) (1-4); it provides two-dimensional maps of the cutaneous temperature distribution, thus providing a dynamic and functional evaluation of thermal properties, in both basal conditions or in response to stimuli $(2,5)$. An altered functional response due to an isometric exercise has been demonstrated as playing an important role in evaluating the variations of cutaneous circulation (6), also in presence of inflammatory diseases such as psoriatic arthritis (PsA) (7). In fact, as we demonstrated in the pilot study (7) and subsequently in our recent work (8), there are significant differences in the thermal behavior of the 20 regions of interest (ROIs) of the dominant hand dorsum, between healthy controls (HCs) and PsA patients after the fulfilment of a mild functional exercise.

The primary aim of this study is to assess qualitatively and quantitatively thermal alterations of patients with rheumatoid arthritis (RA) through fIRI both at baseline and after the same functional exercise we used in the last work (8), and to compare the results with those of HCs.

The secondary goal aims to compare RA thermal values with those of PsA patients previously assessed, and to show the feasibility of fIRI as a new imaging technique for the detection of inflammatory joint dis-
Corresponding author: Alessandra Capo Department of Medicine and Aging Science Dermatologic Clinic, G. d'Annunzio University Chieti, Italy

E-mail: alessandracapo@hotmail.it 
eases such as peripheral PsA and early RA, overcoming powerDoppler-ultrasonography (PWD-US) and magnetic resonance limitations that include cost and time among others.

\section{MATERIALS AND METHODS}

\section{Subjects}

Ten RA patients and 11 HCs were enrolled in a period from January 2014 and April 2015. All participants were matched for sex and age as shown in Table I. All subjects signed their informed consent. HCs had no sign of inflammatory joint diseases and denied any family and personal history of RA or peripheral vascular disorders that could interfere with the fIRI outcome. Moreover, all subjects in the 2 groups were free of any medication and denied any smoking or other habits that could interfere with fIRI measurements (9).

RA diagnosis was performed according to the 2010 ACR/EULAR classification criteria for rheumatoid arthritis (10). All RA patients underwent a PWD-US investigation made with a multiplanar technique according with the EULAR guidelines (European League Against Rheumatism) to assess the severity of the joint disease. The experimental protocol was the same as we used in our recent study on PsA patients measuring thermal values of 20 ROIs of the dominant hand dorsum (8).

Data from RA subjects were then compared with those of the PsA patients we enrolled previously.

\section{Data analysis}

Thermal parameters computed for each ROI for both the entire baseline and recovery periods were:

- average baseline temperature $(\mathrm{Tb})$;
- maximum temperature during the baseline $(\mathrm{Tpb})$;

- minimum temperature during the baseline $(\mathrm{Tmb})$;

- slope of interpolated temperature line during the baseline $(\mathrm{Sb})$;

- average temperature during the recovery (Tr);

- peak temperature during the recovery (Tpr);

- minimum temperature reached after the end of functional exercise (Tmr);

- slope of interpolated temperature line during the recovery $(\mathrm{Sr})$;

- time passed by the end of the exercise to reach Tpr (tp);

- time passed by the end of the exercise to reach $\mathrm{Tmr}$ (tm).

Statistical analysis was processed using a paired student T-test analysis between RA and HCs for each phase, after which we computed the region group average temperature among the subjects.

Before starting the exercise, the subjects were also invited to report on a 10 -value visual analogical scale the cumulative level of pain (P) felt for each of the 14 articular ROIs (11). The relationship between F and $\mathrm{P}$ was assessed through unpaired student $\mathrm{T}$ test analysis between the two groups.

To determine the relationship between joint inflammation, $\mathrm{P}$ and $\mathrm{F}$ in RA patients, Pearson correlation analyses between the group average temperature of the skin regions overlying 14 hand joints and pain intensity were studied. Moreover, correlation analyses among the temperature variables and force based on subject average values were studied through Pearson correlation coefficients. The correlation analysis between performed work during the exercise and joint pain intensity were studied in order to evaluate the effect of pain experienced on

Table I - Demographic data of the participating subjects.

\begin{tabular}{|l|c|c|c|}
\hline Item & HCS & RA & PSA \\
\hline Description & Health control subjects & Rheumatoid patient subjects & Psoriasis patient subjects \\
\hline No. Subjects $=15$ & 11 & 10 & 13 \\
\hline MalelFemale & $5 \backslash 6$ & 317 & 716 \\
\hline Age (Mean \pm SD) (years) & $45 \pm 10.53$ & $59 \pm 15$ & $45 \pm 11$ \\
\hline
\end{tabular}


hand-grasping ability in RA patients. The significant threshold $\alpha$ was set to 0.05 , reduced to 0.0025 and 0.005 after Bonferroni correction for multiple comparisons among group average values and subject average values respectively.

The same analysis was performed to compare data from RA patients with PsA patients previously assessed.

In order to confirm the presence of RA, PWD-US was performed on the ROI on the same day as the thermal recording [Ultrasound Voluson-i (General Electric Medical System, Milwaukee, WI, USA; multifrequency linear probe: 8-16 MHz; Doppler frequency variable up to $8 \mathrm{MHz}$. As requested by International Consensus (12), US parameters evaluated for each ROI were: joint effusion, synovial proliferation, PWD alteration (PWD), joint erosion, tenosynovitis, osteophytosis, median neuropathy. All the evaluations were performed in grayscale mode and through PWD technique. The PWD setting was standardized for all the examinations (pulse repetition frequency: $750 \mathrm{~Hz}$; Doppler frequency:
6-8 MHz). Given the small size sample, no reliable statistical analysis could be performed. Thus only a qualitative analysis and the confirmation of joint involvement is reported.

\section{RESULTS}

The functional exercise was efficient in eliciting appreciable thermal responses in both RA patients and control subjects as shown through a paired t-test for ROIs and for experimental phases, comparing the slope of the temperature trend both during the baseline and the recovery phase. In fact, as expected, during isometric contractions there was a reduction of skin temperature followed by a vasodilation and return to baseline temperature. $\mathrm{Sb}$ and $\mathrm{Sr}$ were statistically different for both $\mathrm{HCs}$ and RA groups $(\mathrm{p}<0.0025)$.

Group average mean and standard deviation values for each parameter are reported in Table II.

For all temperature parameters and pain except $\mathrm{Sb}$, the region group average values

Table II - Group average values mean (standard deviation).

\begin{tabular}{|l|c|c|c|}
\hline Parameter & HCS & PSA & RA \\
\hline Average Baseline $\left(\mathrm{T}_{\mathrm{b}}\right)$ & $32.2(3)$ & $33.2(2)$ & $31(4)$ \\
\hline Maximum Baseline $\left(\mathrm{T}_{\mathrm{pb}}\right)$ & $32.47(3)$ & $33.3(2)$ & $31.25(4)$ \\
\hline Minimum Baseline $\left(\mathrm{T}_{\mathrm{mb}}\right)$ & $32.1(3)$ & $33.1(2)$ & $30.92(4)$ \\
\hline Slope Baseline $\left(\mathrm{S}_{\mathrm{b}}\right)$ & $0.011(0.03)$ & $-0.001(0.01)$ & $0.0097(0.04)$ \\
\hline Average Recovery $\left(\mathrm{T}_{\mathrm{r}}\right)$ & $32.3(3)$ & $33.35(2)$ & $31.5(4)$ \\
\hline Maximum Recovery $\left(\mathrm{T}_{\mathrm{pr}}\right)$ & $32.6(3)$ & $33.7(2)$ & $32(4)$ \\
\hline Time to Maximum Recovery $\left(\mathrm{t}_{\mathrm{pr}}\right) \min$ & $2.17(1.69)$ & $3.45(1.4)$ & $3.74(1.56)$ \\
\hline Minimum Recovery $\left(\mathrm{T}_{\mathrm{mr}}\right)$ & $31.8(3)$ & $32.79(2)$ & $30.8(4)$ \\
\hline Time to Minimum Recovery $\left(\mathrm{t}_{\mathrm{mr}}\right) \mathrm{min}$ & $2.8(0.5)$ & $4.03(0.5)$ & $5.12(0.6)$ \\
\hline Slope Recovery $\left(\mathrm{S}_{\mathrm{r}}\right)$ & $0.009(0.02)$ & $0.02(0.02)$ & $0.03(0.04)$ \\
\hline Pain & $0(0)$ & $3.8(3.6)$ & $3.4(3)$ \\
\hline Force $($ N.s) & $1620(1393)$ & $872(442)$ & $1190(550)$ \\
\hline
\end{tabular}

Abbreviation definitions for the Groups: HCS used for Healthy controls, RHY used for Rheumatoid arthritis and PSA used for Psoriasis arthritis.

Abbreviation definitions for the parameters: $T_{b}$ used for the average baseline temperature, $T_{r}$ used for the average temperature during the recovery phase, $T_{p b}$ used for the maximum temperature during the baseline, $T_{p r}$ used for the peak temperature during the recovery, $t_{p r}$ used for the time to maximum temperature reached after the end of the functional exercise $\left(T_{p r}\right), T_{m b}$ used for the minimum temperature during the baseline, $T_{m r}$ used for the minimum temperature reached after the end of the functional exercise, $t_{m r}$ used for the time to minimum temperature reached near after the end of the functional exercise $\left(\mathrm{T}_{\mathrm{mr}}\right)$, Sb used for the slope of the interpolated temperature line during the baseline, Sr used for the slope of the interpolated temperature line during the recovery, $\mathrm{P}$ used for the pain and $\mathrm{F}$ used for the Force. 
Table III - Correlation between thermal parameters and pain based on region group average values among subjects for RA group.

\begin{tabular}{|c|c|c|c|c|}
\hline \multirow{2}{*}{ Thermal parameter } & \multicolumn{2}{|c|}{ Pain $^{\mathrm{a}}$} & \multicolumn{2}{|c|}{ Force $^{b}$} \\
\hline & $r$ & $\mathrm{P}$-value & $r$ & P-value \\
\hline$T_{b}$ & $0.75^{a k}$ & $<0.0035$ & NA & NA \\
\hline$T_{p b}$ & $0.73^{a x}$ & $<0.0035$ & NA & NA \\
\hline $\mathrm{T}_{\mathrm{mb}}$ & $0.75^{a *}$ & $<0.0035$ & NA & NA \\
\hline$S_{b}$ & $-0.08^{\mathrm{a}}$ & $>0.0035$ & NA & NA \\
\hline$T_{r}$ & $0.86^{a^{k}}$ & $<0.0035$ & NA & NA \\
\hline Tpr & -0.43 & $>0.0035$ & NA & NA \\
\hline $\mathrm{T}_{\mathrm{pr}}$ & $0.85^{a^{k}}$ & $<0.0035$ & NA & NA \\
\hline $\mathrm{T}_{\mathrm{mr}}$ & $0.8^{\mathrm{a}^{\star}}$ & $<0.0035$ & NA & NA \\
\hline$t_{\mathrm{mr}}$ & $-0.7^{a}$ & $>0.0035$ & NA & NA \\
\hline $\mathrm{S}_{\mathrm{r}}$ & $0.37^{a}$ & $>0.0035$ & NA & NA \\
\hline Force & $0.37^{\mathrm{b}}$ & $>0.05$ & NA & NA \\
\hline
\end{tabular}

$\mathrm{T}_{\mathrm{r}}$ : average temperature during the recovery phase; $\mathrm{T}_{\mathrm{mr}}$ : minimum temperature reached after the end of the functional exercise; $T_{p r}$ : peak temperature during the recovery; $t_{m}$ : time passed by the end of the exercise to reach $\left(T_{m r}\right) ; t_{p}$ : time passed by the end of the exercise to reach $\left(T_{p r}\right)$; Sr: slope of the interpolated temperature line during the recovery; $r$ : correlation coefficient; $p$-value: statistical p-value.

*means statistically significant.

${ }^{a}$ means the correlation test based on the region group average values among subjects.

${ }^{b}$ means the correlation test based on the subject group average values among regions. patible with osteophytosis in the studied ROI with significant synovitis in other districts not investigated with fIRI; one patient was observed with median neuropathy.

The comparison between the region group average values of the RA patients versus PsA showed for all temperature parameters and pain except $\mathrm{Sr}$ a statistical significant difference $(\mathrm{p}<0.025)$ (Figures 1 and 2$)$.

The subject group average values among regions did not show any statistical significance between PsA and RA patient groups ( $\mathrm{P}>0.005$ and $>0.05$ for force values).

\section{DISCUSSION AND CONCLUSIONS}

This study tested the feasibility of fIRI to assess the response to a force calibrated as a diagnostic approach for inflammatory joint diseases, such as RA and PsA.

The fundamental hypothesis is that inflammatory-related processes may result in alterations of skin temperature recovery induced by exercise, even in the absence of clinical symptoms.

To this goal, the subjects underwent a mild force stress, which was expected to induce an initial cutaneous vasoconstriction with a temperature reduction, followed by a recovery process with a cutaneous temperature increase $(13,14)$.

RA patients showed higher time to maximum recovery (tpr), Time to minimum recovery (tmr) and Slope Recovery (Sr) was as for PsA patients; thus demonstrating a slower recovery time after exercise compared to HCs.

RA patients showed lower thermal parameters compared to HCs and PsA; thermal IR data showed different patterns in postexercise ROI curves between RA, PsA and HCs. Whereas in HCs the temperature was homogeneous and exponentially increased early after the exercise, in PsA group there was a prolonged delayed re-warming process after the same isometric exercise. It was attributed to a thermal pattern overlaying the inflammatory joint, confirming the results of the previous studies (8). Lower thermal parameters of RA after the exercise are probably due to higher initial cutaneous 

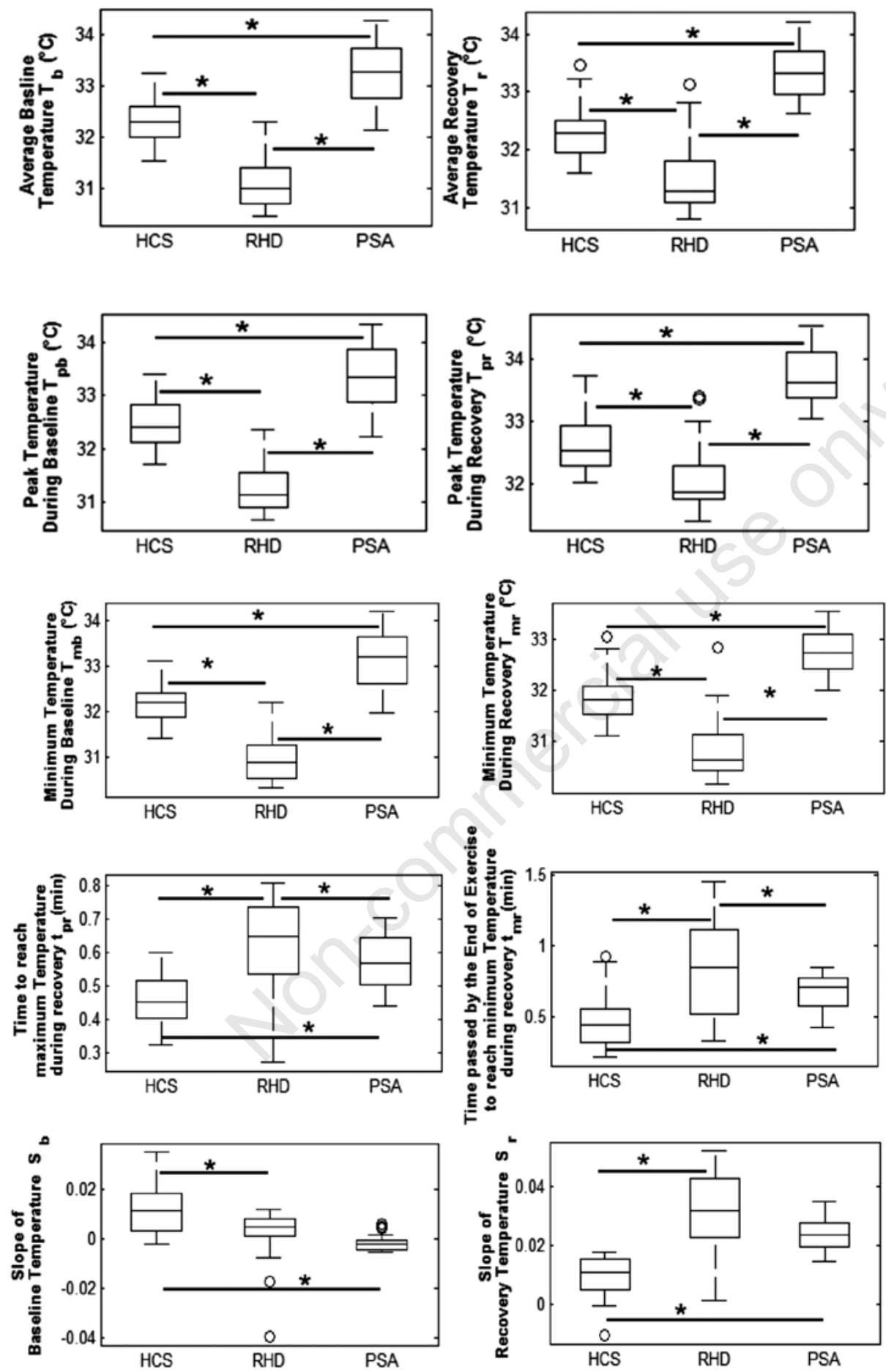

Figure 1 - Comparison of the group average thermal values among groups (Whisker box plot). Median, first, and third quartile, minimum and maximum are reported for each parameter. The box limits represent the upper and lower quartiles; the line inside the box represents the median value. Whiskers extend from each end of the box to minimum and maximum values in the data. The horizontal bars with asterisk connect the groups with significant statistical difference. 


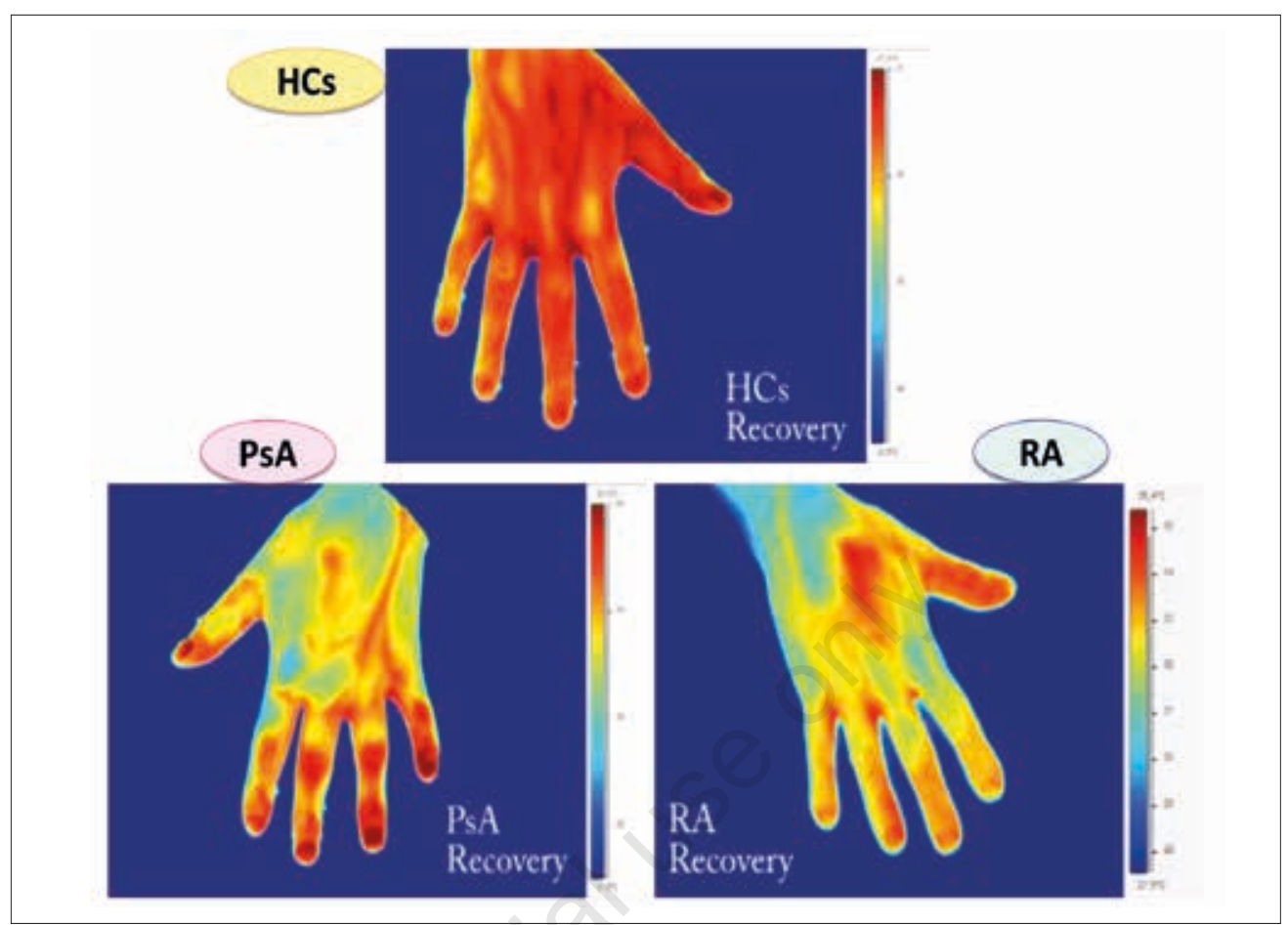

Figure 2 - flR images of recovery phase in HCs, PsA and RA patients.

vasoconstriction related to a major muscle strain. We could speculate that the different thermal behavior shown by RA patients is due to a specific RA inflammatory process: in fact, differently from PsA, in RA joint inflammation leads to a cell proliferation and excessive tissue growth; the synovial cells reproduce at an abnormally fast rate, causing the synovium to thicken, a process called rheumatoid pannus formation. This observation could help to differentiate between RA and PsA.

Force and pain values of the RA group maintained significantly different from HCs, with higher values of pain and lower work performance, suggesting that pain discomfort impaired the ability to perform functional work and induced force reduction (11). This is particularly important in that it explains the finding of work impairment in RA and PsA patients (15).

In conclusion, we have demonstrated the feasibility of fIRI as a new imaging technique for the detection of peripheral RA and PsA. We have also processed a thermoregulatory model based on the automatic control theory and have used its estimat- ed dynamic parameters in order to perform an effective identification of patients affected by RA or PsA (16). A limitation of the fIRI technique could be the difficulty of recording skin temperature in the presence of psoriatic lesions on the dorsum of the hand; moreover, the proposed method is conceived only for detecting MCP and IP joint disease. Further studies are needed to assess the fIR applicability on other sites of joints involvement.

\section{Conflicts of interest: none}

\section{REFERENCES}

1. Merla A, Romani GL, Di Luzio S, et al. Raynaud's Phenomenon: infrared functional imaging applied to diagnosis and drugs effects. Int J Immunopathol Pharmacol. 2002; 15: 41-52.

2. Mariotti A, Grossi G, Amerio P, et al. Finger thermoregulatory model assessing functional impairment in Raynaud phenomenon. Ann Biomed Eng. 2009; 37: 2631-9.

3. Santa Cruz GA, Bertotti J, Marin J, et al. Dynamic infrared imaging of cutaneous melanoma and normal skin in patients treated with BNCT, Appl. Radiat. Isotopes. 2009; 67: S54-8. 
4. Çetingül MP, Herman C. Quantification of the thermal signature of a melanoma lesion. Int $\mathrm{J}$ Thermal Sci. 2011; 50: 421-31.

5. Mariotti A, Di Carlo L, Orlando G, et al. Scrotal thermoregulatory model and assessment of the impairment of scrotal temperature control in varicocele. Ann Biomed Eng. 2011; 39: 664-73.

6. Johnson JM. Exercise in a hot environment: the skin circulation. Scand J Med Sci Sports. 2010; 20: 29-39.

7. Capo A, Merla A, Mattei P, et al. Assessment of psoriatic arthritis by means of functional infrared imaging: a pilot study. Clin Drug Investig. 2013; 33: 59-139.

8. Capo A, Ismail E, Cardone D, et al. Joint functional impairment and thermal alterations in patients with psoriatic arthritis: a thermal imaging study. Microvasc Res. 2015; 102: 86-91.

9. Grossi G, Mariotti A, Di Donato L, et al. Functional infrared imaging of paroxysmal ischemic events in patients with Raynaud's phenomenon. Int J Immunopathol Pharmacol. 2010; 23: 627-32.

10. Kay J, Upchurch KS. ACR/EULAR 2010 rheumatoid arthritis classification criteria.
Rheumatology (Oxford). 2012; 51 Suppl 6: vi5-9.

11. Ilowite NT, Walco GA, Pochaczevsky R. Assessment of pain in patients with juvenile rheumatoid arthritis: relation between pain intensity and degree of joint inflammation. Ann Rheum Dis. 1992; 51: 343-6.

12. Wakefield RJ, D’Agostino MA, Iagnocco AM, et al. The OMERACT Ultrasound Group: status of current activities and research directions. J Rheumatol. 2007; 34: 848-51.

13. Shibasaki M, Secher NH, Johnson JM, Crandall CG. Central command and the cutaneous vascular response to isometric exercise in heated humans. J Physiol. 2005; 565: 667-73.

14. Johnson JM, Park MK. Effect of upright exercise on threshold for cutaneous vasodilation and sweating. J Appl Physiol. 1981; 50: 814-8.

15. Ayala F, Sampogna F, Romano GV, et al. The impact of psoriasis on work-related problems: a multicenter cross-sectional survey. J Eur Acad Dermatol Venereol. 2014; 28: 1623-32.

16. Ismail E, Capo A, Amerio P, Merla A. Functional-thermoregulatory model for the differential diagnosis of psoriatic arthritis. BioMed Engine OnLine. 2014; 13: 162. 